\title{
Self-healing Interconnects with Nearly Plastic Stretching of Repairs
}

\author{
Amit Kumar, ${ }^{1}$ Virendra Parab, ${ }^{1}$ Arindan Handu, ${ }^{1}$ Li Ding, ${ }^{2}$ Pushkaraj Joshi, ${ }^{2}$ Chen Jiang, ${ }^{2}$ and \\ Sanjiv Sambandan ${ }^{1,2, *}$ \\ ${ }^{1}$ Department of Instrumentation and Applied Physics, Indian Institute of Science, Bangalore 560012, India \\ ${ }^{2}$ Department of Engineering, University of Cambridge, Cambridge CB3OFF, United Kingdom
}

(Received 28 August 2018; revised manuscript received 6 December 2018; published 29 January 2019)

\begin{abstract}
Flexible electronic systems such as roll-up displays and wearable devices promise exciting possibilities that could change the way humans interact with the environment. However, they suffer from poor reliability of interconnects and devices. Interconnects on flex are prone to open-circuit failures due to mechanical stress, electrostatic discharge, and environmental degradation. Passive approaches such as the use of stretchable conductors and novel geometries improve their response to mechanical stress but cannot salvage the interconnect if a fault were to occur. Active approaches using self-healing techniques can repair a fault and have been demonstrated with use of methods that use relatively rare materials, change conventional interconnect-fabrication processes, address only faults due to mechanical stress, or do not permit stretching. In this work we discuss a self-healing technique that overcomes these limitations and demonstrate heals having metallic conductivity and nearly plastic stretchability. This is achieved by dispersion of conductive particles in an insulating fluid encapsulated over the interconnect. Healing is automatically triggered by the electric field appearing in the open gap of a failed interconnect, irrespective of the cause of failure. The field polarizes the conductive particles, causing them aggregate and chain up to bridge the gap and repair the fault. Using dispersions of copper microspheres in silicone oil, we show self-healing interconnects with the stretchable heal having conductivity of about $5 \times 10^{5} \mathrm{~S} / \mathrm{m}$ and allowing strains from 12 to 60. Previously, stretchable interconnects used materials other than copper. Here we effectively show self-healing, stretchable copper. This work promises high-speed, self-healing, and stretchable interconnects on flex, thereby improving system reliability.
\end{abstract}

DOI: 10.1103/PhysRevApplied.11.014057

\section{INTRODUCTION}

Electronics on flex offer aesthetic and functional advantages such as the possibility of roll-to-roll manufacturing [1], use of organic metals and semiconductors [2-4], sensors and actuators on flex [4-7], energy sources [8-10], and wearable devices for health diagnostics [11-14]. However, these advantages are accompanied by poor reliability of devices [15] and interconnects [16]. Interconnects on flex experience mechanical forces due to stretching, bending, and thermal stress and unexpected current surges due to electrostatic discharge and environment-related degradation. As a result, open interconnect faults occurring during system operation are common.

Both passive and active techniques have been investigated to address this problem. Passive approaches tailor interconnect geometries [4,17-25] and materials [19,26-39] to increase mechanical flexibility. While they increase tolerance to mechanical-stress-driven faults, they do not prevent faults occurring due to other mechanisms.

\footnotetext{
*ss698@cam.ac.uk
}

Moreover, they do not provide a means to reestablish connectivity if an open fault does occur. On the other hand, active techniques offer online repair of an open fault. Self-healing of interconnects using conductive polymers [40,41], liquid metals [42-44], embedding of capsules of conductive inks in interconnects that spill the ink on fracture [45-47], controller-based impedance modulation [48], Janus particles driven by differences in hydrophobicities [49], ionic gels [50], and electric-field-driven interconnect restructuring using dispersions has been investigated [51-55]. While these approaches have been shown to be very effective, the techniques have some shortcomings as they use relatively rare materials (e.g., Ga, In), change conventional interconnect-fabrication processes, address only mechanical-stress-related faults, or do not permit stretchability.

An ideal solution to increasing open-fault tolerance of interconnects on flex would have the following attributes. First, it would be a self-driven repair mechanism. Second, it would permit a heal having near metallic conductivity. Third, it would permit a heal having high stretchability. Fourth, the repair mechanism would be activated irrespective of the cause of the fault. 
This work provides an affirmative answer to all four points. Here we use a dispersion of electrically conductive particles in an insulating fluid that is contained and isolated over each interconnect as shown in Fig. 1(a). On the occurrence of an open-circuit fault in a currentcarrying interconnect, the electric field appearing across the open gap polarizes the conductive particles of the dispersion inside the gap. The polarized particles experience dipole-dipole attractive forces and eventually chain up and sinter to create a bridge across the gap, thereby healing the fault; therefore, the repair mechanism is independent of the cause the fault. This mechanism also permits near plastic stretchability [Fig. 1(b)]. When the substrate is stretched, the heal momentarily breaks. This reestablishes the field in this newly formed break, polarizes locally available free particles, and encourages them to fill the gap to reestablish connectivity. The heal therefore stretches by increasing the number of particles constituting the chain, thereby making this a unique approach to stretchable self-healing. Figure 1(c) demonstrates this concept using a series resistor-light-emitting diode (LED) circuit on a polydimethylsiloxane (PDMS) substrate and a dispersion of copper microspheres $(80 \mathrm{mg} / \mathrm{ml}$; radius $5 \mu \mathrm{m})$ in silicone oil (see Appendix A for the dispersion-preparation method). For demonstrations, the interconnect used is a single strand $(100 \mu \mathrm{m})$ of a multistrand wire. Further, a well containing the dispersion is created over an exposed region of the interconnect and an open fault is deliberately introduced in this region. The entire sample is mechanically clamped to the translation stage for stretching. When an external voltage is applied, the LED does not initially light up due to the open fault. However, the electric field across the gap activates the self-healing mechanism, resulting in the LED eventually lighting up. When the entire system is stretched, the connectivity is maintained (with intermittent breaks) due to the heal increasing its length. The events occurring inside the well are shown in Fig. 1(c).

Henceforth we discuss the mechanics of self-healing and a key process of a self-driven sintering. This sintering is important to achieve mechanical flexibility in the heal. We characterize the self-healing mechanism and the response of the heal to stretching. All experiments in this work use a dispersion of copper microspheres in silicone oil (see Appendix A for the dispersion-preparation method).

\section{MECHANICS OF HEALING WITH SINTERING}

To study the self-healing mechanism, an open-circuit fault is emulated with use of the test bed shown in Fig. 2(a). A dispersion of copper microspheres (radius $5 \mu \mathrm{m}$ ) in
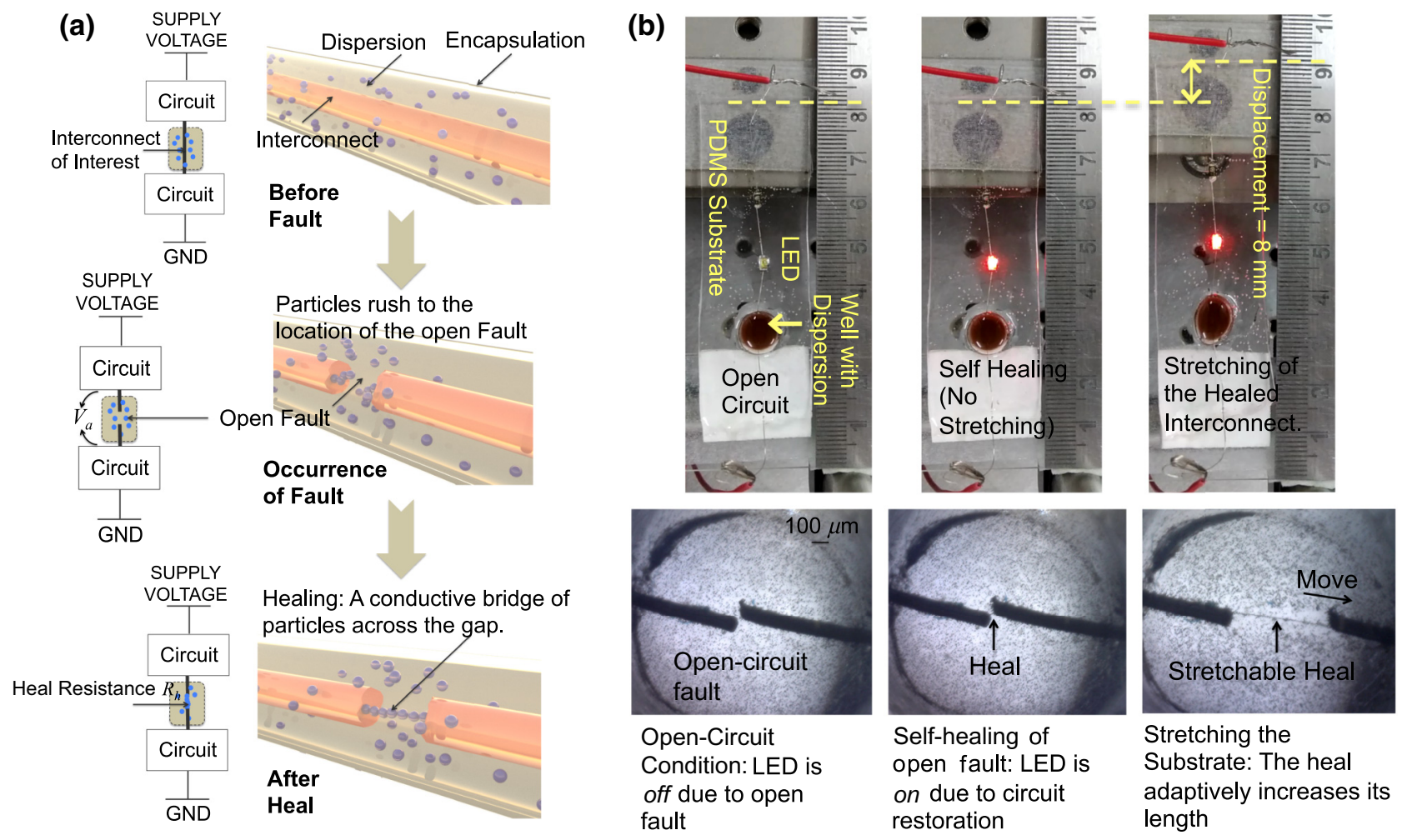

FIG. 1. (a) The self-healing mechanism. (b) Demonstration of stretchable self-healing with the heal adaptively increasing in length to accommodate the stretching. The dispersion is contained in a well and over an interconnect with an artificially induced open fault. Initially, the fault is healed. On stretching of the substrate, the heal stretches while maintaining electrical connectivity. The events occurring inside the well are also shown. See also Videos 1 and 2 in Appendix B. GND, ground. 
(a)

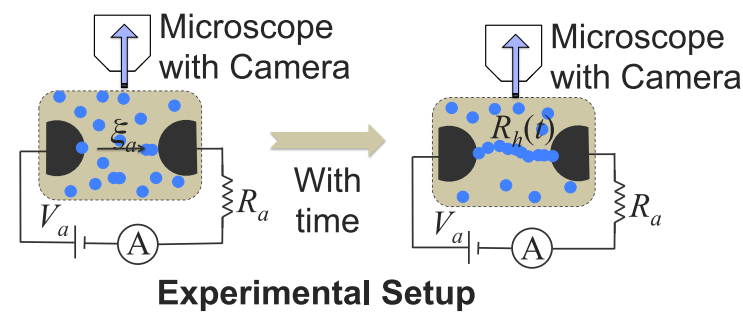

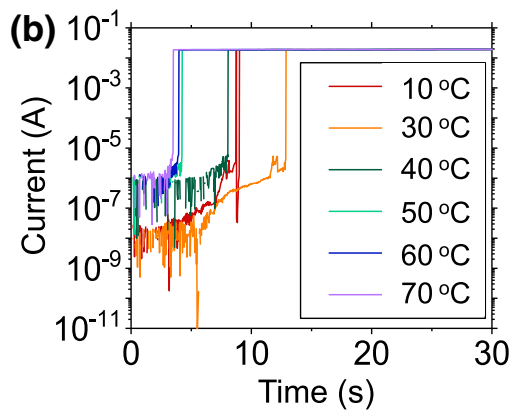

(c)

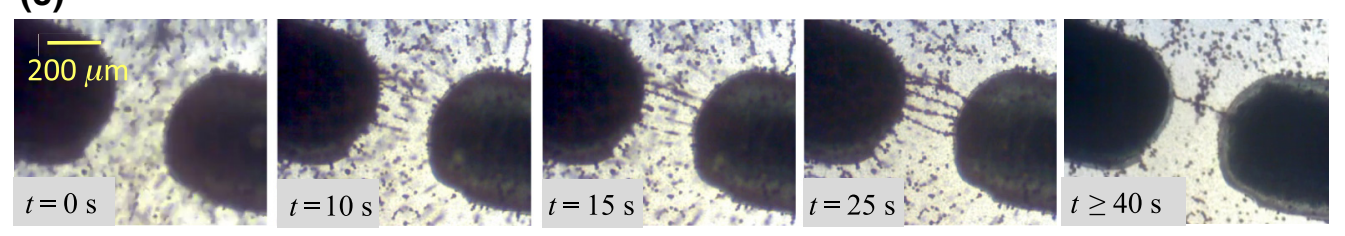

(d)

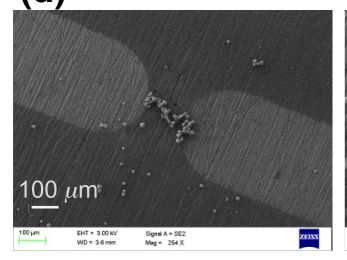

(e)

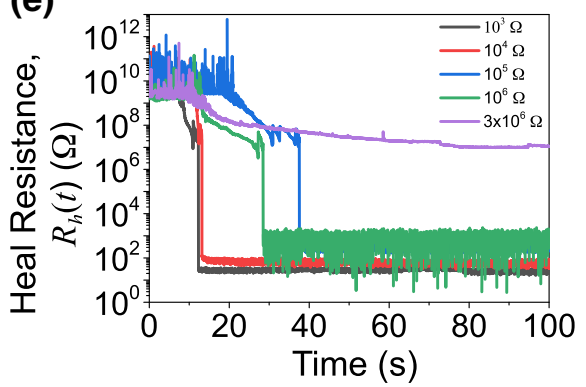

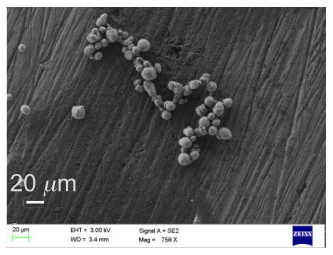
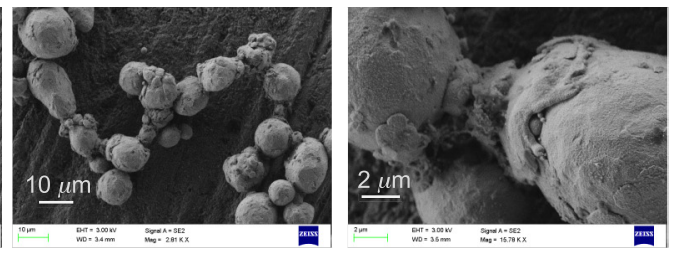

(f)

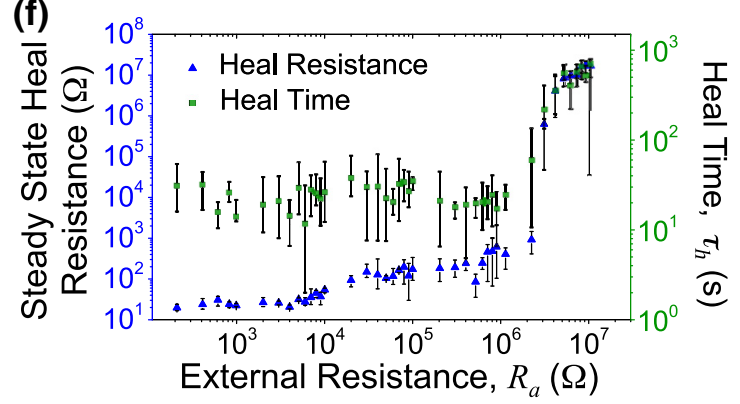

FIG. 2. (a) Experimental setup for the study of the mechanics of self-healing. (b) Current versus time for several self-healing experiments conducted with dispersions at different temperatures. At higher temperatures, the viscosity of the fluid is low and the healing is quicker. The sudden jump in current is indicative of the sintering of the heal. (c) Photographs of the self-healing process (see also Video 3 in Appendix B). (d) SEM images of the sintered heal. (e) Transients of the heal resistance, $R_{h}(t)$, during healing for different external resistances, $R_{a}$. (f) The heal time and steady-state heal resistance are dependent on $R_{a}$ [56].

silicone oil (see Appendix A for the dispersion-preparation method) is contained in a $200-\mu \mathrm{m}$-wide gap between two electrodes. This gap mimics the open fault, while the electrodes represent the two ends of the disconnected interconnect. External resistors are placed in series to emulate the terminal impedances of the interconnect. The total external impedance is $R_{a} \sim 4.4 \mathrm{k} \Omega$. A Keithley 2410 source meter unit is used as the source of the voltage, $V_{a}$, and to measure the dynamics of the current through the circuit throughout the experiment. Figure 2(b) shows the typical observed dynamics of the current during the healing process for experiments performed with dispersions at different temperatures. The temperature influences the viscosity of the fluid and therefore the dynamics of healing.
In real applications, the occurrence of an open fault in a current-carrying interconnect would itself result in the electric field and initiate the healing process. However, for the purpose of this study, an electric field, $\xi_{a}$, is artificially created across the gap by application of an external voltage, $V_{a}$. The field strength is chosen with a view toward the possible application of self-healing for printed electronic thin-film-transistor circuits on flex. In these systems, the operating voltages are typically $5-15 \mathrm{~V}$, and open faults causing $10-\mu \mathrm{m}$ gaps [56] would result in fields of approximately $0.5-1.5 \mathrm{~V} / \mu \mathrm{m}$. With this in mind, a field of $\xi_{a} \approx 0.4 \mathrm{~V} / \mu \mathrm{m}$ is created across the gap by application of an external voltage $V_{a}=80 \mathrm{~V}$ to the circuit in Fig. 2(a). 
On experiencing this field the conductive particles of the dispersion in the gap are polarized. As shown in the sequence of photographs in Fig. 2(c), the induced dipole moment in the copper particles results in them chaining up due to dipole-dipole attraction and forming chains of loosely held linear clusters $(t=25 \mathrm{~s})$. Once these chains span the gap and bridge the two electrodes, the current through the interconnect is established to a small extent. This current causes Joule heating. For certain conductive particles (e.g., copper), this heating results in a weak sintering of the particles, thereby leading to reduction of the heal resistance with time. This sintered bridge constitutes the heal. After sintering, the current through the interconnect increases rapidly, leading to the restoration of electrical connectivity. Figure 2(d) shows SEM images of the sintered copper particles in the bridge (see Appendix A).

The total healing time, $\tau_{h}$, is the sum of two components: the time taken for the formation of the chains of electrostatically held particles and the time taken for sintering and formation of the single-wire-like bridge. The first component (i.e., the time for the formation of chains) is estimated by our equating the dipole-dipole attractive force that drives the mechanism of chain formation with the opposing force of viscous drag experienced by the particles moving through the fluid. This time can be shown to scale as approximately $\left(\eta_{f} / \epsilon_{f}\right)\left(x_{p} / r_{p}\right)^{5} \xi_{a}^{-2}$, with $\eta_{f}$ being the dynamic viscosity of the fluid, $\epsilon_{f}$ the permittivity of the fluid, and $x_{p}$ the average distance between the conductive particles in a homogeneous dispersion (see Appendix A). The second component (i.e., the dynamics of sintering) is governed by a positive-feedback mechanism that can be qualitatively described as follows: On the formation of the multiple chains of loosely held particles across the gap, the current through the interconnect distributes itself through these chains. However, minor differences in the resistance of each chain result in the current through the chains being different, such that one of the chains, typically the shortest, carries the maximum current and heats the quickest. This results in quicker sintering and the resistance being lowered further, which in turn increases the current and the heating through the chain. This results in the restoration and stabilization of the current through the interconnect. If the time-dependent resistance of the heal is $R_{h}(t)$, the voltage drop across the gap would be $V_{a} R_{h}(t) /\left(R_{h}(t)+R_{a}\right)$ and the rate of increase in temperature of the bridge would be proportional to $V_{a}^{2} R_{h}(t) /\left(R_{h}(t)+R_{a}\right)^{2}$. This heating leads to the sintering and the reduction of $R_{h}(t)$ with time. The dependence of the dynamics of $R_{h}(t)$ on $R_{a}$ is shown in Fig. 2(e). The dependence of the healing time and the steady-state heal resistance on $R_{a}$ is shown in Fig. 2(f). For successful self-healing, the interconnect must have low terminal impedance (i.e., it must be a current-carrying interconnect).

\section{SELF-HEALING WITH STRETCHING}

Figure 3(a) shows the experimental setup to study the response of the heal to one-dimensional (1D) stretching [56]. To emulate the electrodes and the encapsulated dispersion as envisioned in Fig. 1(a), a half-open microchannel (100- $\mu \mathrm{m}$ width and $500-\mu \mathrm{m}$ depth) is molded on the surface of a PDMS substrate. The channel is then filled (from one side via capillary action) with the dispersion of copper microspheres in silicone oil. Two wires $(100-\mu \mathrm{m}$ diameter) are inserted into the channel from the two ends to form the two electrodes. One of the wires is attached to a precision translation stage (Thor Labs BSC 101 SIN 40833095) that enables it to move back and forth in the channel with a precise velocity. The surface is finally capped with Kapton tape. The tape confines the dispersion and restricts the movement of the electrode to one dimension. The external circuit is similar to that in Fig. 2(a) but with $R_{a}=220 \mathrm{k} \Omega$.

To begin, the spacing between the electrodes is adjusted to $40 \mu \mathrm{m}$ (initial position) to emulate an open fault. Healing is first achieved at this initial position, and a sintered bridge is allowed to form across the 40- $\mu \mathrm{m}$ gap (time $t=$ $0 \mathrm{~s}$ ). When the current through the interconnect is stable, the electrodes are moved apart at a constant relative velocity of $u=5 \mu \mathrm{m} / \mathrm{s}$ (after initial acceleration from rest). Figure 3(b) illustrates a sequence of photographs from the experiment. The heal appeared to "stretch" to accommodate the movement of the electrodes (see Videos 4 and 5 in Appendix B). The dynamics of the current through the circuit is continuously recorded throughout the experiment [Fig. 3(c)]. The shaded subplot is an enlarged view of the time span from 50 to $100 \mathrm{~s}$. Despite intermittent breaks, the connectivity is repeatedly restored until around $900 \mathrm{~s}$. Beyond this, the connectivity is permanently lost.

Following from the discussion of Fig. 1(b), this phenomenon of persistent restoration of electrical conductivity can be explained as follows. Initially, with the electrodes separated by an open gap, the particle chain bridges the gap, sinters, and restores electrical connectivity. When the electrodes begin to move apart due to stretching, the sintered chain momentarily experiences large stress and breaks. However, a strong electric field is reestablished in this new gap that is formed due to the break. The presence of the electric field once again initiates the self-healing mechanism and involves locally available free particles in the healing by polarizing them. The new particles that fill the gap soon sinter to become a permanent part of the heal [Fig. 3(d)]. The heal therefore responds to stretching by adding more particles to its length.

The repeated drops and increases in current [Fig. 3(c)] also corroborate this explanation. However, this repeated restoration of electrical conductivity does not last forever. If the local concentration of particles become too low or if the velocity of separation, $u$, is too high, electrical 
(a)

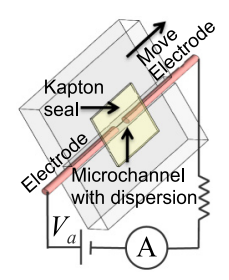

(b)

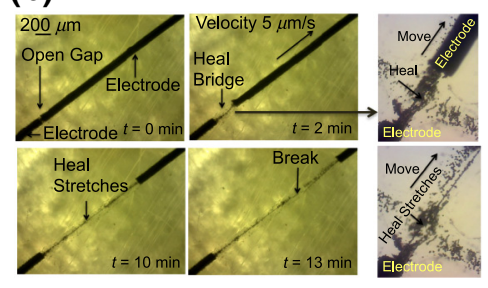

(e)
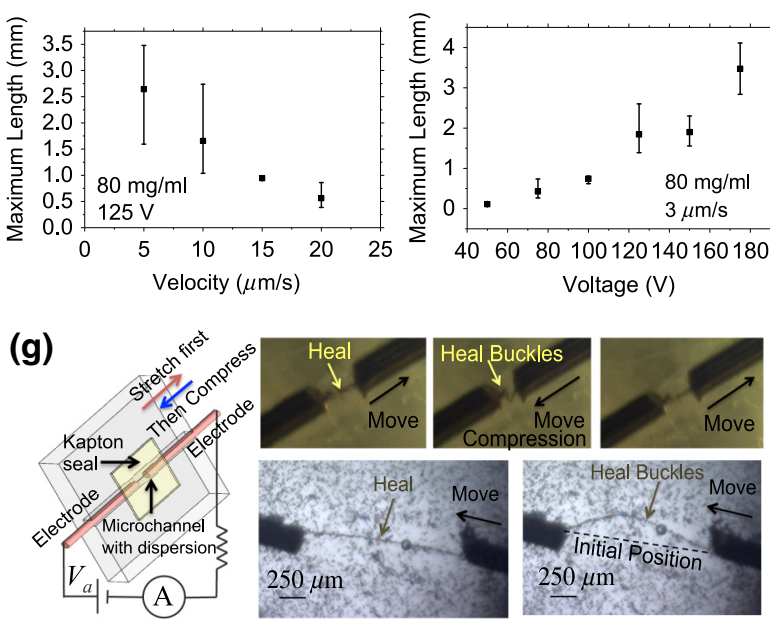

(c)
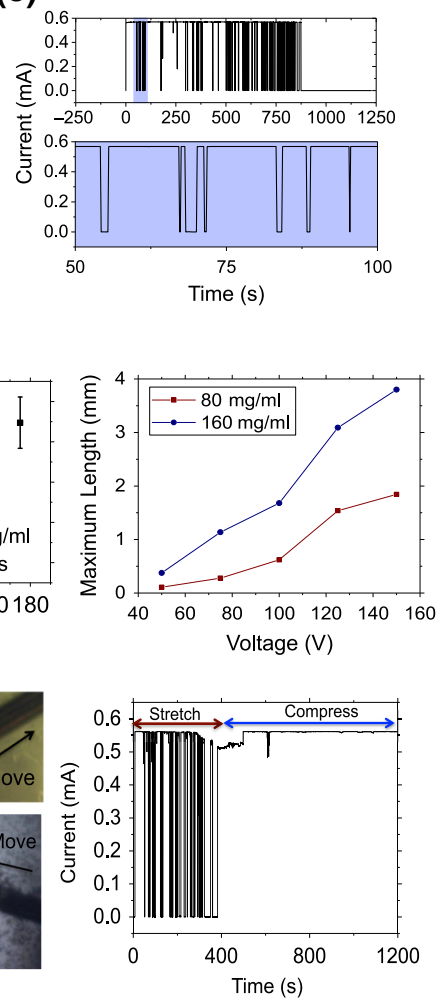

(d)

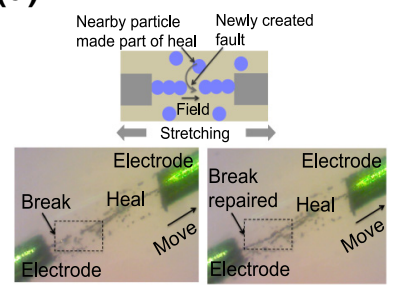

(f)
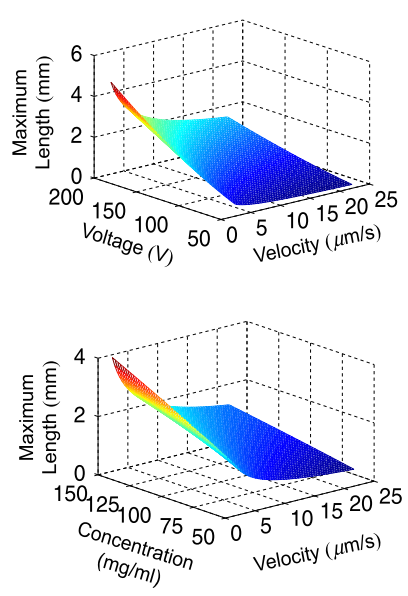

FIG. 3. (a) Experimental setup for the study of the impact of stretching on the heal. (b) Photographs from experiments demonstrating the stretchability of the heal. At $t=0$, the open gap of length $40 \mu \mathrm{m}$ is healed. The electrodes are then moved apart at a constant velocity of $5 \mu \mathrm{m} / \mathrm{s}$. (c) In response to stretching, the heal adaptively increases its length by involving more particles in the healing process. (d) Measurements of current versus time during the stretching [56]. (e) Maximum stretchable length, $\Delta l_{\max }$, as a function of the stretching velocity, $u$, applied voltage, $V_{a}$, and dispersion concentration [56]. (f) Prediction of $\Delta l_{\max }$ as a function of $u, V_{a}$, and dispersion concentration based on Eq. (1). (g) Buckling of the heal in response to compression and the typical dynamics of the current through the heal in response to stretching and subsequent compression. See also Videos 4-7 in Appendix B.

connectivity is permanently lost (here at around $900 \mathrm{~s}$ ). In the former case, there are no particles available to fill in the gaps occurring in the expanding heal. In the latter case, the increasing distance between the electrodes rapidly weakens the field and weakens the dipole moment and the driving force for healing. Therefore, if the break is not filled in by a nearby particle immediately, permanent restoration becomes increasingly harder. The maximum length, $\Delta l_{\max }$, to which the heal can stretch without permanent loss of electrical connectivity depends on $V_{a}, u$, and the dispersion concentration. As shown in Fig. 3e, $\Delta l_{\max }$ reduces with an increase in $u$, increases with an increase in $V_{a}$, and increases with an increase in dispersion concentration.

To quantify $\Delta l_{\max }$, we consider the case where there exists a sintered heal across the open gap between two electrodes. At time $t=0$, the electrodes are moved apart with a constant velocity $u$. The stress in the sintered heal causing a fracture at some location in the heal and this new gap grows in length as $u t$ and the field across the gap reduces as approximately $V_{a} / u t$ (ignoring the nonuniformity of field). This field is responsible for reactivating self-healing and filling in this gap with the locally available particles. However, if this field drops below a threshold electric field $\xi_{\text {th }}$, the dipole-dipole attractive forces become too weak to overcome the static friction and the Brownian motion and conductivity is not restored. The time taken for the electric field to drop below $\xi_{\text {th }}, \tau_{\text {th }}$ scales as approximately $V_{a} /\left(u \xi_{\text {th }}\right)$. If the nearest free particles is at a distance of approximately $x_{p}$, the time constant for the particle to arrive at the gap can be shown to be approximately $\left(\eta_{f} / \epsilon_{f}\right)\left(x_{p} / r_{p}\right)^{5} u^{2} t^{2} / V_{a}^{2}$. If the time for sintering is ignored, this time constant defines the total repair time of the newly formed gap. Therefore for the heal to stretch successfully by the addition of more particles along its length, this repair time must be less than or equal to $\tau_{\text {th }}$. Clearly as time $t$ increases, this condition is less likely to be satisfied. Therefore, the heal can stretch till equality is obtained in time constants, and therefore

$$
\Delta l_{\max } \propto\left(\epsilon_{f} / \eta_{f}\right)^{1 / 2}\left(r_{p} / x_{p}\right)^{5 / 2}\left(u \xi_{\mathrm{th}}\right)^{-1 / 2} V_{a}^{3 / 2}
$$


As an example, a dispersion of $r_{p}=5 \mu \mathrm{m}$ particles of concentration $80 \mathrm{mg} / \mathrm{ml}$ in silicone oil of kinematic viscosity $300 \mathrm{cSt}$ implies $\eta_{f}=0.28 \mathrm{~kg} / \mathrm{ms}, \epsilon_{f}=20 \times 10^{-12}$ $\mathrm{F} / \mathrm{m}$, and $x_{p}=38.8 \mu \mathrm{m}$. If $\xi_{\text {th }}=0.04 \mathrm{~V} / \mu \mathrm{m}$, the theoretical value for constant of proportionality in Eq. (1) can be shown to be 0.05 . The best fit to data is obtained by our defining the constant of proportionality in Eq. (1) as 0.06, resulting in the predicted dependence of $\Delta l_{\max }$ on $V_{a}$ and $u$ as shown in Fig. 3(f). On the other hand, for a constant voltage of $125 \mathrm{~V}$, the dependence of $\Delta l_{\max }$ on the dispersion concentration (in weight per volume) and $u$ is shown in Fig. 3(f).

We next consider the case of 1D oscillations (i.e., 1D stretching followed by 1D compression of the heal) as described by Fig. 3(g). After being stretched and subsequently compressed, the heal grows in length to accommodate the stretching and subsequently buckles to accommodate compression, all the while maintaining electrical connectivity. Figure 3(g) shows the buckling in the heal during compression observed during different experiments. The typical dynamics of the current through the heal in response to stretching and subsequent compression is also shown.

For experiments on two-dimensional stretching, a $5 \times$ $5 \mathrm{~mm}^{2} \times 2 \mathrm{~mm}$ (depth) well in PDMS is used to contain the dispersion. Single-strand wires of $100 \mu \mathrm{m}$ are slightly bent at the ends and immersed in the dispersion to form the electrodes. One of the wires is connected to two approximately orthogonally aligned translation stages (Holmarc MTS 3760 for axial control and Thor Labs BSC 101 SIN 40833095 for tangential control) [56]. The external circuit is similar to that in Fig. 3(a). At the start of the experiment, the electrodes are placed $40 \mu \mathrm{m}$ apart and the voltage is applied, resulting in a heal. Once the current stabilizes, the electrodes are moved apart axially, allowing the heal to stretch. After a $1.15-\mathrm{mm}$ displacement, axial translation is stopped and tangential translation is activated. The images in Fig. 4(a) illustrate the impact of tangential movement. The heal therefore experiences two kinds of forces. The first is the stress due to axial stretching. The second is the distributed loading along its length due to viscous drag experienced by the heal as it moves through the fluid during tangential movement. Except for intermittent loss of conductivity, this two-dimensional movement does not disrupt the electrical connectivity between the electrodes. However, for large displacements, the heal is permanently fractured, resulting in a loss of conductivity. The current is plotted as a function of time during the experiment in Fig. 4(b). The maximum subtended angle between the initial position of the heal to the final position (when the heal fractures permanently) is plotted as a function of the velocity of movement along the tangential direction in Fig. 4(c). Figure 4(c) also shows the maximum displacement along the tangential direction before failure. (a)

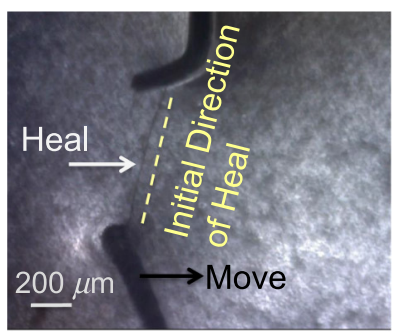

(b)

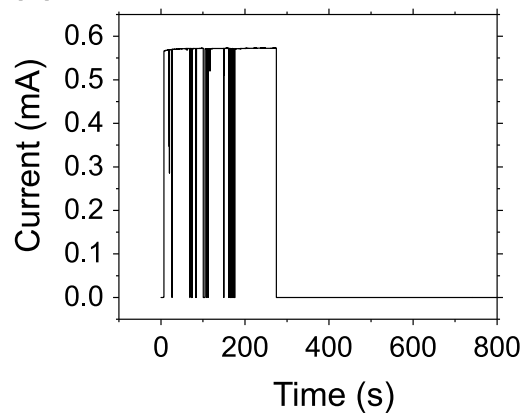

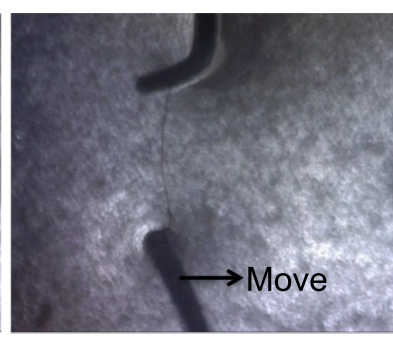
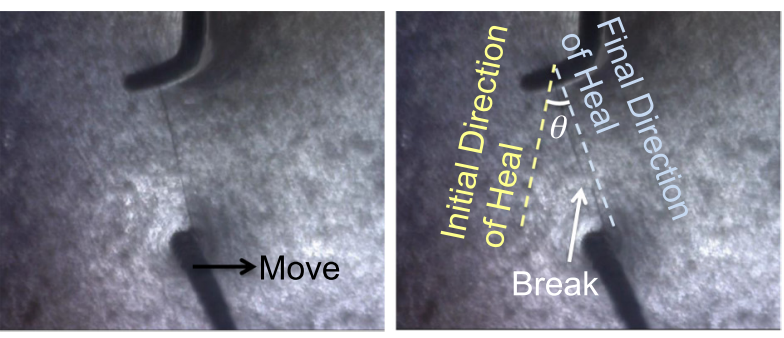

(c)

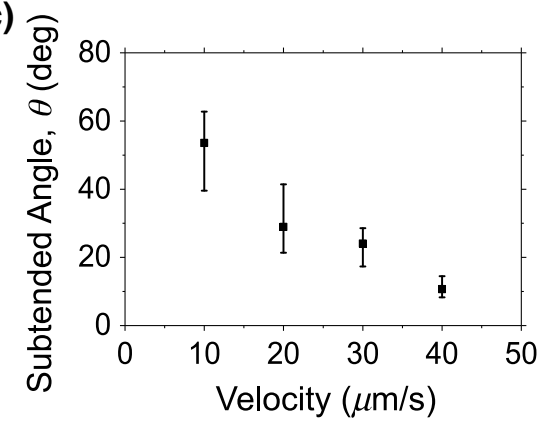

FIG. 4. (a) Demonstration of stretching in two dimensions. The electrodes are moved axially and then tangentially. The initial direction of the heal across the open gap is shown before the onset of tangential movement. The final direction of the heal is shown, defining the moment the chain breaks and cannot stretch any further. The maximum angle subtended between the initial and the final direction is $\theta$. (b) Plot of the typical current transients during the two-dimensional movement [56]. (c) The subtended angle $\theta$ as a function of the velocity and the maximum displacement length as a function of the velocity [56]. See also Video 8 in Appendix B. 
(a)

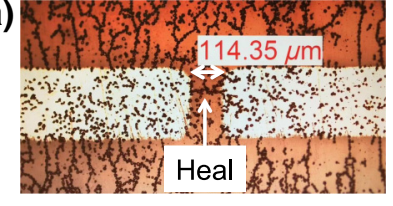

(b)

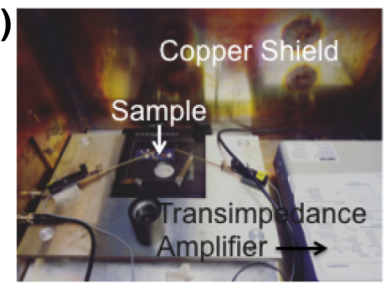

(c)

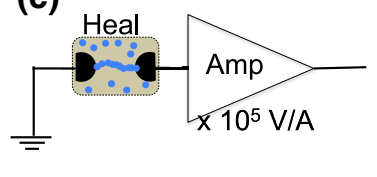

(d)

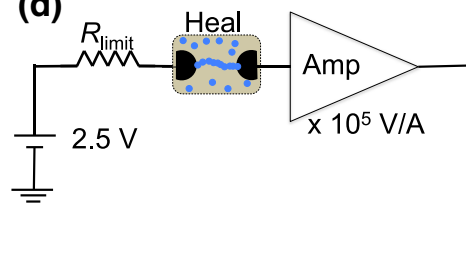

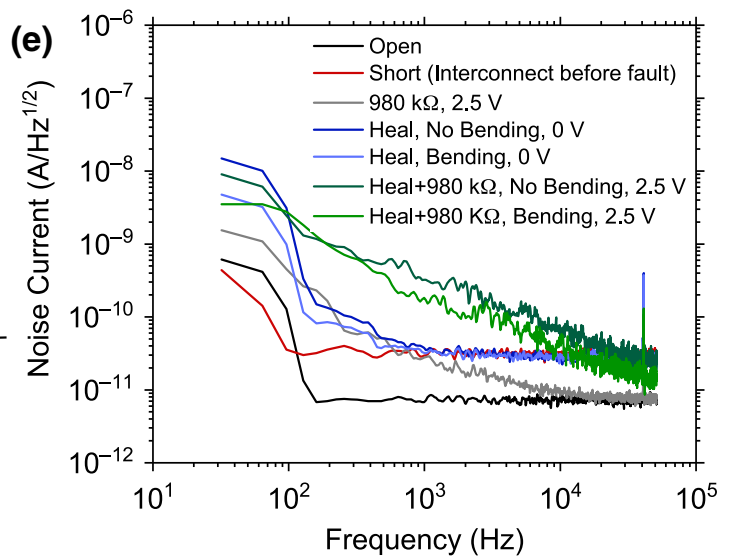

FIG. 5. (a) Self-healing with printed silver interconnects on polyethylene naphthalate substrate. (b) Noise-measurement setup. The sample is placed in a copper-shield box. A battery-powered transimpedance amplifier is used to measure the noise current. (c) Noise measurement without direct current. (d) Noise measurement with direct current. (e) Measured noise current for several cases.

The noise performance of the heal is also studied (Fig. 5). The healing is performed with silver interconnects printed on polyethylene naphthalate, which is a common substrate used for flexible electronic applications [Fig. 5(a)]. The experimental setup used for the measurements is described in Appendix A and is shown in Fig. 5(b). Noise measurements of the heal are conducted with the two circuits shown in Fig. 5(c) (no direct current) and Fig. 5(d) (with direct current) in flat and bent (1-cm bending radius) conditions. Noise measurements in the absence of a direct current are expected to show the presence of thermal noise alone. The thermal-noise current is expected to have a power of $4 k T / R$, with $R$ being the resistance, $k$ the Boltzmann constant, and $T$ the temperature. First, the measurements are calibrated by our measuring the noise in open- and short-circuit conditions. The noise current measured in the open-circuit condition yielded the noise of the measurement setup. The noise current measured in the short-circuit condition showed the noise of the interconnect itself. For noise-current measurements in the presence of a direct current, a dc voltage supplied by a $2.5-\mathrm{V}$ battery is used. Moreover, since the transimpedance amplifier has a direct-current limit and since the heal resistance is very small (about $100 \Omega$ ), an external resistor is also used in series with the heal resistance. The noise due to the external resistor is measured for calibration. All results are shown in Fig. 5(e). Noise measurements in the absence of direct current are expected to show thermal noise. The measured noise current is about $0.1 \mathrm{nA} / \sqrt{\mathrm{Hz}}$. Measurements with the $\mathrm{dc}$ battery in series are expected to show flicker noise as well. The corner frequency is a little above $50 \mathrm{kHz}$. While the above-mentioned noise measurements are performed when the sample is at rest, it is clear from Fig. 3 that there appear larger current fluctuations during stretching due to the periodic breaks and heals. If these fluctuations were to be considered noise, then they would be the largest source of noise.

\section{DISCUSSION}

This work presents the possibility of realizing interconnects with the ability to automatically heal an open-circuit failure with the heal having an ability to stretch. This is achieved by the use of a rather simple system - dispersion of conductive particles in an insulating fluid. If an open fault were to occur in a current-carrying interconnect, the resulting electric field in the gap would initiate the selfhealing mechanism by polarizing the conductive particles located in the gap, resulting in them chaining up (dipoledipole attraction) to eventually form a bridge across the gap. The repair mechanism is therefore truly self-driven (i.e. it self-activates and self-terminates). The occurrence of the fault creates the electric field that initiates the self-healing mechanism. The healing mechanism restores conductivity by forming a sintered chain of particles (the heal) across the open gap. Once the gap is short-circuited by the heal, the field disappears, discouraging further chain formation and stopping the mechanism. The healing mechanism is akin to blood clotting where the various species in the blood rush to the site of injury to participate in clotting.

The key feature of the healing mechanism is the possibility of compressing and stretching the heal without losing electrical connectivity. A requirement to achieve this is the sintering of the particles constituting the bridge. This too is self-driven due to Joule heating governed by positive feedback for as long as $R_{h}(t)>R_{a}$. Sintering makes the heal behave like a conductive thread or chain. In compression, this chain could accommodate compression by buckling appropriately. In tension (stretching), the chain would break. Since the chain segments on either side of this break are conductive, the electric field would be 
reestablished in this newly formed gap, thus reactivating the healing by encouraging locally available particles to fill this gap and complete the chain. Thus the sintered chain would accommodate stretching by effectively adding more particles to its length. This is a fundamental difference compared with the use of conductive materials that stretch due to a low modulus of elasticity.

For the sake of comparison with other stretchable conductors, it is useful to model the effective mechanical properties of the heal. With use of dispersions of copper microspheres $(80 \mathrm{mg} / \mathrm{ml})$ in silicone oil, for $V_{a}=125$ $\mathrm{V}$, the maximum displacement ranged from $0.5 \mathrm{~mm}$ (for $u=20 \mu \mathrm{m} / \mathrm{s}$ ) to $2.5 \mathrm{~mm}$ (for $u=5 \mu \mathrm{m} / \mathrm{s}$ ). Since the initial separation of the electrodes is $40 \mu \mathrm{m}$, this is equivalent to a minimum strain of 12.5 to a maximum strain of 60.25 depending on the strain rate. Although there is an influence of the strain rate, the stress dynamics due to an increase in strain is not like that of a viscoelastic material. Instead, the averaged effective behavior of the heal is to respond to the increase in strain by adding more particles to increase its length and with no increase in average stress. Therefore it is more appropriate to model this behavior as plastic with the rupture point dependent on the strain rate.

For complete integration of the self-healing mechanism with integrated circuits on flex, three major problems need to be overcome. The first is with regard to the encapsulation and techniques for packaging of the dispersion with high-density interconnects in the manner shown in Fig. 1(a). The solution to this problem may lie in borrowing ideas from inkjet-printed electronics and microfluidics. These technologies are compatible with flexible electronics and would permit the controlled dispensing and packaging of the dispersion. The second problem is with regard to scaling down. All experiments described in this paper use interconnects and open gaps of width of approximately $100 \mu \mathrm{m}$. This permits the use of dispersions having metallic particles of diameter of approximately $10 \mu \mathrm{m}$. However, thin-film-transistor-based integrated circuits on flex typically use interconnects of width approximately $100 \mu \mathrm{m}$, which would result in open faults of widths of the same length scale. For this study to scale down, the dispersion must now use particles with diameter of approximately $1 \mu \mathrm{m}$. This poses problems with the stability of the dispersion as well as impacting the time taken to heal due to much-weaker dipole-dipole forces and a more-influential Brownian motion. This problem can be overcome by increase of the dispersion concentration. However, such an approach spills over to the third major problem, which is with regard to cross talk. As the dispersion concentration is increased and as the dispersion heads toward becoming a conductive fluid, the significantly three-dimensional feature of the interconnects due to the encapsulation would result in significant cross talk between two adjacent interconnects, thereby limiting the frequency response. Therefore, the use of a very high dispersion concentration is not ideal and this parameter needs to be optimized.

Nevertheless, this approach to self-healing is versatile and has the potential for application across a wide class of systems, such as flexible and wearable electronics to commercial printed circuit boards. This versatility stems from two features. First, the approach does not use rare materials (e.g., Ga-In, graphene) but instead uses a dispersion of copper particles in a common insulating fluid (transformer oil). Second, the approach does not change the conventional material and processes used for interconnect fabrication. Instead, the concept can be implemented as an add-on feature requiring additional fabrication processes if selfhealing is desired. These features permit interconnects with the ability to self-heal and stretch on flexible substrates, thereby increasing interconnect reliability significantly.

\section{CONCLUSION}

This work demonstrates the possibility of self-healing interconnects with the ability to stretch significantly. This is demonstrated with dispersions of copper microspheres in silicone oil. Heals having near metallic conductivity of approximately $5 \times 10^{5} \mathrm{~S} / \mathrm{m}$ and stretchability with strains from 12 to 60 depending on the strain rate are demonstrated. Previously, stretchable interconnects used materials other than copper. Here we effectively show self-healing, stretchable copper. This work promises highspeed, self-healing, and stretchable interconnects on flex, thereby increasing system reliability.

\section{ACKNOWLEDGMENTS}

This work was funded by the EPSRC (Grant No. RG92121) and DST IMPRINT (Grant No. 7969). A.K. and V.P. contributed equally to this work. S.S. thanks the DBT India-Cambridge Lectureship Program for permitting a joint appointment between the Indian Institute of Science and the University of Cambridge.

\section{APPENDIX A: MATERIALS AND METHODS}

\section{Dispersion preparation}

For the experiments discussed in this work, dispersions of copper microspheres (Alfa Aesar product no. 042689) in silicone oil (S. D. Fine-Chem Limited, product no. 25072) are used. The copper microspheres have an average radius of $5 \mu \mathrm{m}$ and the oil has a kinematic viscosity of $300 \mathrm{cSt}$. Dispersions of various concentrations are estimated in milligrams per milliliter and are prepared by our measuring the weight of copper microspheres to be dispersed in a known volume of silicone oil. The optimum concentration is found to lie between 50 and $150 \mathrm{mg} / \mathrm{ml}$. The dispersion is sonicated for $1 \mathrm{~h}$ at $80^{\circ} \mathrm{C}$ followed by mechanical stirring to achieve the required homogeneity. No special stabilization techniques are used. At room temperature, it 
is observed that the viscosity of the fluid aided the preparation of relatively stable and homogeneous dispersions that were sufficiently suitable for the applications discussed in this work.

\section{Sample preparation for SEM imaging}

For SEM images [Fig. 2(d)], the experiment is first performed as described in Fig. 2 on a printed circuit board. The current versus time is constantly measured. Thirty seconds after the current is restored and stabilized (indicative of sintering), the circuit is disconnected. The regions between the electrodes now contain the sintered heal immersed in silicone oil. To obtain SEM images, this region is repeatedly rinsed with isopropyl alcohol to remove the oil. The sample is then dried and the section of the printed circuit board containing the bridge is transferred to a SEM stub for imaging. Conductive carbon tape is used to short-circuit the electrodes (and therefore the heal) to the SEM stub for good imaging.

\section{Dynamics of chain formation}

The driving force for chain formation is the dipoledipole attraction between the polarized particles. The dipole-dipole attractive force is defined by $F_{p-p}=$ $P^{2} / \epsilon_{f} x^{4}$, with $P=4 \pi \beta r_{p}^{3} \epsilon_{f} \xi_{a}$ being the dipole moment, $x$ the center-to-center distance between two polarized particles, $\epsilon_{f}$ the permittivity, $\xi_{a}$ the electric field, and $\beta$ the Clausius-Mossotti factor. For dc electric fields and for particles having a conductivity much larger that that of the fluid, $\beta \approx 1$. This driving force of dipole-dipole attraction is balanced by the viscous drag, $F_{p-f}$ experienced by the particle in the fluid. This drag is given by Stokes law as $F_{p-f}=6 \pi \eta_{f} r_{p}(d x / d t)$. The dynamics defining the movement and aggregation of the particles is given by our equating these two forces: $F_{p-p}=F_{p-f}$. If $x_{p}$ is the initial average distance between the particles in the dispersion (i.e., $x=x_{p}$ at $t=0$ ), the solution to the differential equation implies that the time taken for two particles to meet each other $\left(x=2 r_{p}\right)$ scales as approximately $\left(\eta_{f} / \epsilon_{f}\right)\left(x_{p} / r_{p}\right)^{5}\left(1 / \xi_{a}^{2}\right)$.

\section{Noise-current-measurement setup}

The sample is placed in a copper box and the interconnect is probed to measure the noise current. Since bending experiments are to be performed, silver interconnects on a flexible substrate (polyethylene naphthalate) are used for self-healing experiments. Noise signals are first amplified by a preamplifier located inside the copper box. The preamplifier (AMETEK model 5182) is powered by batteries. The gain is set to $10^{5} \mathrm{~V} / \mathrm{A}$. The output of the preamplifier is sent via coaxial cables to a dynamic signal analyzer (Agilent 35670A). The signal analyzer measures the noise spectrum in a bandwidth from $32 \mathrm{~Hz}$ to $51.2 \mathrm{kHz}$.

\section{APPENDIX B: VIDEOS}

The following videos correspond to the experiments shown in the figures. Videos 1 and 2 correspond to Fig. 1 and demonstrates an application of self healing with stretching. Video 3 corresponds to Fig. 2 and is used to understand the physics of self healing. Videos $4-6$ correspond to Fig. 3 and are used to understand the physics and mechanism of stretching of the repair. Video 7 also corresponds to Fig. 3 and describes the behaviour of the heal upon buckling. Video 8 corresponds to Fig. 4 and describes the behaviour of the heal between two electrodes when the electrodes are moved orthogonally to the direction of the heal.

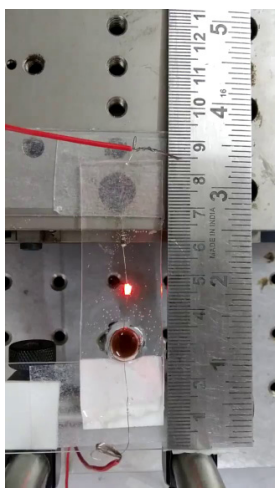

VIDEO 1. Demonstration of stretchable self-healing with the heal adaptively increasing in length to accommodate the stretching.

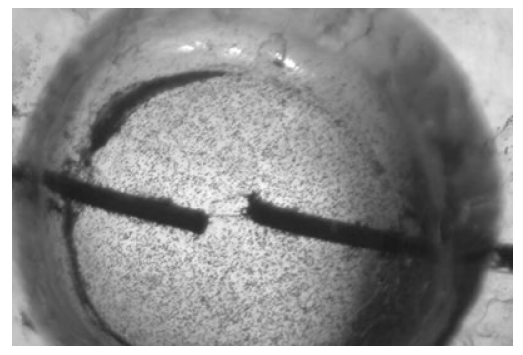

VIDEO 2. Enlargement of events inside the well for the demonstration of self-healing in Video 1.

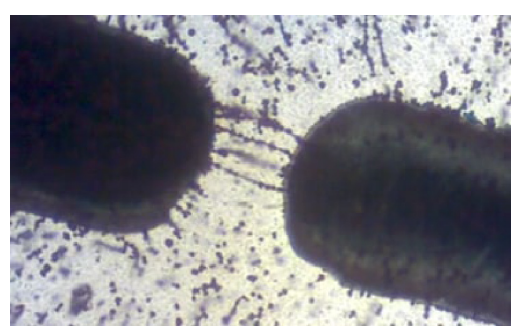

VIDEO 3. Mechanism of self-healing. 


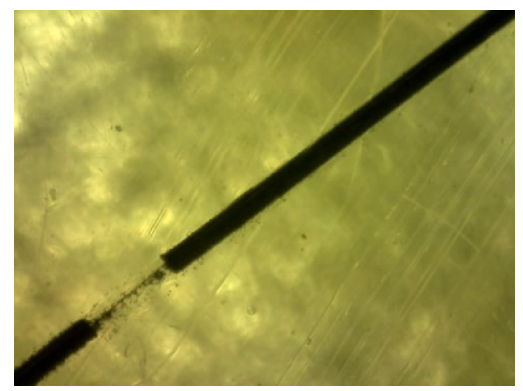

VIDEO 4. Stretchable self-healing.

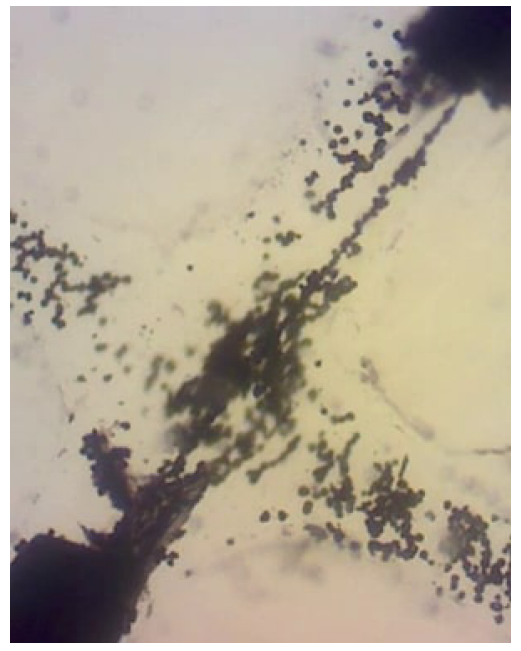

VIDEO 5. Detailed view of the stretching of the heal.

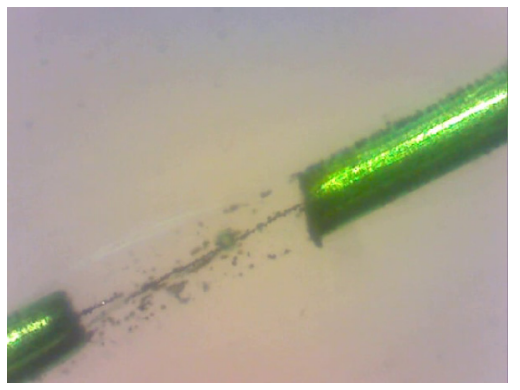

VIDEO 6. Mechanism of stretching of the heal. The intermittent breaks during stretching result in a large field tat polarizes a nearby particle. This particle fills in the gap to repair the break.

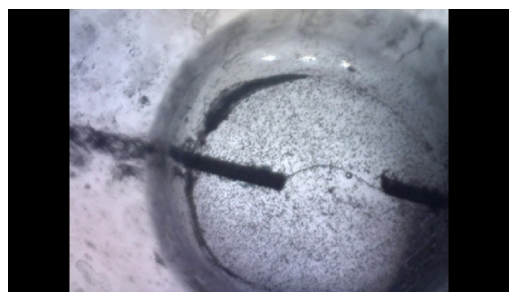

VIDEO 7. Buckling of the heal during compression.

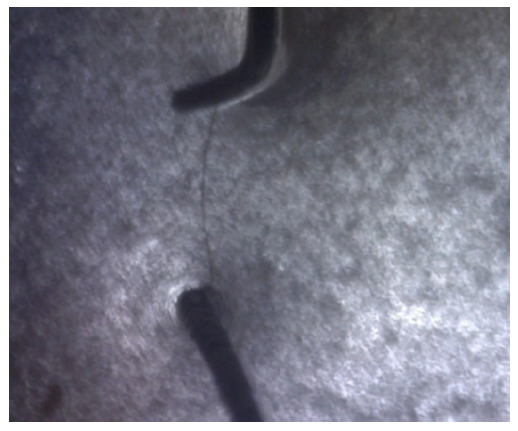

VIDEO 8. Two-dimensional stretching.

[1] W. Wong and A. Salleo Fabrication on Web by Roll-to-Roll Processing (Springer, New York, 2009).

[2] A. J. Heeger, N. S. Sariciftci, and E. B. Namdas, Semiconducting and Metallic Polymers (Oxford University Press, New York, 2010).

[3] R. D. Jansen-van Vuuren, A. Armin, A. K. Pandey, P. L. Burn, and P. Meredith, Organic photodiodes: The future of full color detection and image sensing, Adv. Mater. 28, 4766 (2016).

[4] J. Rogers, T. Someya, and Y. Huang, Materials and mechanics for stretchable electronics, Science 327, 1603 (2010).

[5] T. N. Ng, W. S. Wong, M. L. Chabinyc, S. Sambandan, and R. A. Street, Flexible image sensor array with bulk heterojunction organic photodiode, Appl. Phys. Lett. 92, 213303 (2008).

[6] K. J. Yu, Z. Yan, M. Han, and J. Rogers, Inorganic semiconducting materials for flexible and stretchable electronics, Flexible Electron. 1, 4 (2017).

[7] T. Someya, Building bionic skin, IEEE Spectr. 50, 50 (2013).

[8] A. M. Gaikwad, D. A. Steingart, T. Ng, D. E. Schwartz, and G. L. Whiting, A flexible high potential printed battery for powering printed electronics, Appl. Phys. Letts. 102, 233302 (2013).

[9] P. Kodali, A. Krishna, R. Varun, M. Prasad, and S. Sambandan, Segmented electrodes for piezoelectric energy harvesters, IEEE Electron. Dev. Letts. 35, 485 (2014).

[10] P. Kodali, G. Saravanavel, and S. Sambandan, Crumpling for energy: Modeling generated power from the crumpling of polymer piezoelectric foils for wearable electronics, Flexible Printed Electron. 2, 035005 (2017).

[11] H. Guo, M.-H. Yeh, Y.-C. Lai, Y. Zi, C. Wu, Z. Wen, C. Hu, and Z. L. Wang, All-in-one shape-adaptive self-charging power package for wearable electronics, ACS Nano 10, 10580 (2016)

[12] Y. Takeda, K. Hayasaka, R. Shiwaku, K. Yokosawa, T. Shiba, M. Mamada, D. Kumaki, K. Fukuda, and S. Tokito, Fabrication of ultra-thin printed organic TFT CMOS logic circuits optimized for low-voltage wearable sensor applications, Sci. Rep. 6, 25714 (2016).

[13] S. Nakata, T. Arie, S. Akita, and K. Takei, Wearable, flexible, and multifunctional healthcare device with an ISFET 
chemical sensor for simultaneous sweat $\mathrm{pH}$ and skin temperature monitoring, ACS Sens. 2, 443 (2017).

[14] J. Rivnay, S. Inal, A. Salleo, R. M. Owens, M. Berggren, and G. G. Malliaras, Organic electrochemical transistors, Nat. Rev. Mater. 3, 17086 (2018).

[15] F. R. Libsch and J. Kanicki, Bias-stress-induced stretchedexponential time dependence of charge injection and trapping in amorphous silicon thin film transistors, Appl. Phys. Lett. 62, 1286 (1993).

[16] S. Sambandan, R. B. Apte, W. S. Wong, R. Lujan, M. Young, B. Russo, S. Ready, and R. A. Street, Defect identification in large area electronic backplanes, IEEE J. Disp. Technol. 5, 27 (2009).

[17] J. Mertens, R. W. Bowman, J. C. Willis, A. Robinson, D. Cotton, R. White, K. A. Seffen, and J. J. Baumberg, Scalable Microaccordion Mesh for Deformable and Stretchable Metallic Films, Phys. Rev. Appl. 4, 044006 (2015).

[18] B. Huyghe, H. Rogier, J. Vanfleteren, and F. Axisa, Design and manufacturing of stretchable high frequency interconnects, IEEE Trans. Adv. Packag. 31, 802 (2008).

[19] W. Dang, V. Vinciguerra, L. Lorenzelli, and R. Dahiya, Printable stretchable interconnects, Flexible Printed Electron. 2, 013003 (2017).

[20] D.-H. Kim, Z. Liu, Y.-S. Kim, J. Wu, J. Song, H.-S. Kim, Y. Huang, K.-C. Hwang, Y. Zhang, and J. A. Rogers, Optimized structural designs for stretchable silicon integrated circuits, Small 24, 2841 (2009).

[21] R. Verplancke, F. Bossuyt, D. Cuypers, and J. Vanfleteren, Thin-film stretchable electronics technology based on meandering interconnections: Fabrication and mechanical performance, J. Micromech. Microeng. 22, 015002 (2012).

[22] G. Eda, G. Fanchini, and M. Chhowalla, Large-area ultrathin films of reduced graphene oxide as a transparent and flexible electronic material, Nat. Nanotechnol. 3, 270 (2008).

[23] R. Verplancke, F. Bossuyt, D. Cuypers, and J. Vanfleteren, Thin-film stretchable electronics technology based on meandering interconnections: Fabrication and mechanical performance, J. Micromech. Microeng. 22, 015002 (2012).

[24] D.-H. Kim, N. Lu, R. Ma, Y.-S. Kim, R.-H. Kim, S. Wong, J. Wu, S. M. Wu, H. Tao, and J. Rogers, Epidermal electronics, Science 333, 838 (2011).

[25] S. P. Lacour, J. Jones, S. Wagner, T. Li, and Z. Suo, Stretchable interconnects for elastic electronic surfaces, Proc. IEEE 11, 1459 (2005).

[26] S. Lacour, G. Courtine, and J. Guck, Materials and technologies for soft implantable neuroprostheses, Nat. Rev. Mater. 1, 16063 (2016).

[27] H. C. Jung, J. H. Moon, D. H. Baek, J. H. Lee, Y. Y. Choi, J. S. Hong, and S. H. Lee, Cnt/pdms composite flexible dry electrodes for long-term ecg monitoring, IEEE Trans. Biomed. Eng. 59, 1472 (2012).

[28] C.-X. Liu and J.-W. Choi, Patterning conductive pdms nanocomposite in an elastomer using microcontact printing, J. Micromech. Microeng. 19, 085019 (2009).

[29] T. Araki, M. Nogi, K. Suganuma, M. Kogure, and O. Kirihara, Printable and stretchable conductive wirings comprising silver flakes and elastomers, IEEE Electron Device Lett. 32, 1424 (2011).

[30] L. Jiajie, T. Kwing, and P. Qibing, A water-based silvernanowire screen-print ink for the fabrication of stretchable conductors and wearable thin-film transistors, Adv. Mater. 28, 5986 (2016).

[31] V. Martinez, F. Stauffer, M. O. Adagunodo, C. Forro, J. Voros, and A. Larmagnac, Stretchable silver nanowireelastomer composite microelectrodes with tailored electrical properties, ACS Appl. Mater. Interfaces 7, 13467 (2015).

[32] K. Tybrandt, F. Stauffer, and J. Voros, Multilayer patterning of high resolution intrinsically stretchable electronics, Sci. Rep. 6, 25641 (2016).

[33] X. Feng and Z. Yong, Highly conductive and stretchable silver nanowire conductors, Adv. Mater. 24, 5117 (2012).

[34] K. K. Kim, S. Hong, H. M. Cho, J. Lee, Y. D. Suh, J. Ham, and S. H. Ko, Highly sensitive and stretchable multidimensional strain sensor with prestrained anisotropic metal nanowire percolation networks, Nano Lett. 15, 5240 (2015).

[35] Y. Sungryul, N. Xiaofan, Y. Zhibin, H. Weili, B. Paul, and P. Qibing, Compliant silver nanowire-polymer composite electrodes for bistable large strain actuation, Adv. Mater. 24, 1321 (2012).

[36] L. Jinhwan, L. Phillip, L. H. Beom, H. Sukjoon, L. Inhwa, Y. Junyeob, L. S. Seob, K. Taek-Soo, L. Dongjin, and K. S. Hwan, Room-temperature nanosoldering of a very long metal nanowire network by conducting-polymerassisted joining for a flexible touch-panel application, Adv. Funct. Mater. 23, 4171 (2013).

[37] S. Choi, J. Park, W. Hyun, J. Kim, J. Kim, Y. B. Lee, C. Song, H. J. Hwang, J. H. Kim, T. Hyeon, and D.-H. Kim, Stretchable heater using ligand-exchanged silver nanowire nanocomposite for wearable articular thermotherapy, ACS Nano 9, 6626 (2015).

[38] Y. Tang, S. Gong, Y. Chen, L. W. Yap, and W. Cheng, Manufacturable conducting rubber ambers and stretchable conductors from copper nanowire aerogel monoliths, ACS Nano 8, 5707 (2014).

[39] B. W. An, E.-J. Gwak, K. Kim, Y.-C. Kim, J. Jang, J.-Y. Kim, and J.-U. Park, Stretchable, transparent electrodes as wearable heaters using nanotrough networks of metallic glasses with superior mechanical properties and thermal stability, Nano Lett. 16, 471 (2016).

[40] J. Y. Oh, S. Rondeau-Gagn, Y.-C. Chiu, A. Chortos, F. Lissel, G.-J. N. Wang, B. C. Schroeder, T. Kurosawa, J. Lopez, T. Katsumata, J. Xu, C. Zhu, X. Gu, W.-G. Bae, Y. Kim, L. Jin, J. W. Chung, J. B.-H. Tok, and Z. Bao, Intrinsically stretchable and healable semiconducting polymer for organic transistors, Nature 539, 411 (2016).

[41] K. A. Williams, A. J. Boydston, and C. W. Bielawski, Towards electrically conductive, self-healing materials, J. Mater. Chem. 4, 359 (2007).

[42] E. Palleau, S. Reece, M. E. Desai, S. C. Smith, and M. D. Dickey, Self-healing stretchable wires for reconfigurable circuit wiring and 3D microfluidics, Adv. Mater. 25, 1589 (2013). 
[43] G. Li, X. Wu, and D.-W. Lee, A galinstan-based inkjet printing system for highly stretchable electronics with selfhealing capability, Lab Chip 16, 1366 (2016).

[44] E. J. Markvicka, M. D. Bartlett, X. Huang, and C. Majidi, An autonomously electrically self-healing liquid metalelastomer composite for robust soft-matter robotics and electronics, Nat. Mater. 17, 618 (2018).

[45] B. Blaiszik, S. Kramer, M. Grady, D. Mcllroy, J. Moore, N. Sottos, and S. White, Autonomic restoration of electrical conductivity, Adv. Mater. 24, 398 (2011).

[46] M. M. Caruso, S. R. Schelkopf, A. C. Jackson, A. M. Landry, P. V. Braun, and J. S. Moore, Microcapsules containing suspensions of carbon nanotubes, J. Mater. Chem. 19, 6093 (2009).

[47] S. Odom, M. M. Caruso, A. Finke, Alex M. Prokup, J. Ritchey, J. Leonard, S. R. White, N. R. Sottos, and J. S. Moore, Restoration of conductivity with TTF-TCNQ charge-transfer salts, Adv. Funct. Mater. 20, 1721 (2010).

[48] S. B. Bowers, K. Sengupta, K. Dasgupta, B. Parker, and A. Hajimiri, Integrated self-healing for $\mathrm{mm}$-wave power amplifiers, IEEE Trans. Microwave Theory Tech. 61, 1301 (2013).

[49] X. Yong, E. J. Crabb, N. M. Moellers, and A. C. Balazs, Self-healing vesicles deposit lipid-coated Janus particles into nanoscopic trenches, Langmuir 29, 16066 (2013).
[50] Y. Cao, T. G. Morrissey, E. Acome, S. I. Allec, B. M. Wong, C. Keplinger, and C. Wang, A transparent, self healing, highly stretchable ionic conductor, Adv. Mater. 29, 1605099 (2016).

[51] J.-B. Fleury, D. Pires, and Y. Galerne, Self-Connected 3D Architecture of Microwires, Phys. Rev. Letts. 103, 267801 (2009).

[52] S. Sambandan, Self repair in circuits-automating open fault repair in integrated circuits using field-induced aggregation of carbon nanotubes, IEEE Trans. Elec. Dev. 59, 1773 (2012).

[53] A. Nair, K. Raghunandan, V. Yaswant, S. Pillai, and S. Sambandan, Maze solving automatons for self-healing of open interconnects: Modular add-on for circuit boards, Appl. Phys. Lett. 106, 123103 (2015).

[54] V. Parab, L. Ding, A. Kumar, V. Yaswant, and S. Sambandan, Self-healing interconnects for reliable flexible electronics, ECS Trans. 85, 825 (2018).

[55] V. Yaswanth, A. Kumar, and S. Sambandan, Self healing of open circuit faults: With active re-configurability and mimicry of synaptic plasticity, Appl. Phys. Letts. 109, 024101 (2016).

[56] See Supplemental Material at http://link.aps.org/supple mental/10.1103/PhysRevApplied.11.014057 for details on open faults in printed interconnects and dispersion preparation and plots of experimental data. 